\title{
A cesium beam atomic clock using laser optical pumping. Preliminary tests (*)
}

\author{
M. Arditi \\ Institut d'Electronique Fondamentale, Université Paris-Sud, \\ Bâtiment 220, F91405 Orsay Cedex, France
}

and J. L. Picqué

Laboratoire Aimé-Cotton (**),

Centre National de la Recherche Scientifique, Bâtiment 505, F91405 Orsay Cedex, France.

(Reçu le 3 juin 1980, accepté le 26 juin 1980)

\begin{abstract}
Résumé. - Un résonateur microonde passif à jet de césium utilisant le pompage optique par un laser accordable continu monomode (ici, un laser à $\mathrm{GaAs}$ ) a été réalisé. La différence de population entre les niveaux de la transition hyperfine de l'état fondamental de ${ }^{133} \mathrm{Cs}(\mathrm{à} 9192,631 \ldots \mathrm{MHz}$ ) est produite par pompage optique à l'aide du laser à GaAs; la résonance microonde est détectée par la modification de l'intensité de la fluorescence induite par le même laser. Des tests préliminaires du fonctionnement de l'appareil en horloge atomique ont été effectués.
\end{abstract}

\begin{abstract}
A passive microwave cesium beam resonator utilizing optical pumping with a C.W. narrowband tunable laser (here, a GaAs laser) has been realized. A population difference between the levels of the ground state hyperfine transition of ${ }^{133} \mathrm{Cs}$ (at $9192.631 \ldots \mathrm{MHz}$ ) is achieved by optical pumping with the GaAs laser; the microwave resonance is detected through a change in the intensity of the fluorescence induced by the same laser. Preliminary tests of operation of the apparatus as an atomic clock have been performed.
\end{abstract}

The hyperfine field insensitive transition $F=3$, $m_{F}=0 \leftrightarrow F=4, m_{F}=0$ of the ground state of the cesium atom ${ }^{133} \mathrm{Cs}$ (at $9192631770 \mathrm{~Hz}$ by definition) is now the basis for the determination of the unit of time. Usually, this microwave transition is detected with the Rabi-Ramsey atomic beam magnetic resonance method: The preparation and analysis of the magnetic state of the atoms are achieved by inhomogeneous magnetic fields, and the signal is obtained by ionization of the atoms on a Langmuir-Taylor detector [1]. The possibility of replacing in this method the deflecting magnets by optical interaction regions [2] has been proved already in an experiment involving a beam of ${ }^{87} \mathrm{Rb}$ atoms optically pumped with $\mathrm{a}^{85} \mathrm{Rb}$ spectral lamp [3]. However, more recently, the advent of tunable lasers has made possible efficient hyperfine pumping of a cesium atomic beam [4], which allows one to expect a sensitive optical detection of the clock transition. Optically pumped cesium beam fre-

$\left(^{*}\right)$ Version anglaise d'une Note présentée à l'Académie des Sciences par Mr. A. Kastler [C.R. Hebd. Séan. Acad. Sci. (1980)].

$\left.{ }^{* *}\right)$ Laboratoire Associé à l'Université Paris-Sud. quency standards should have, in principle, some advantages [4] over the present primary frequency standard [1]. One of them could be a reduction (and a more precise measurement) of the frequency bias connected with the cavity phase shifts seen by atoms having different trajectories.

The laser used in the present experiment is a semiconductor GaAs diode laser, delivering a few milliwatts of power under C.W. single-mode operation. The laser is temperature-stabilized around $25 \mathrm{~K}$ in a cryostat, and the injected current is about $200 \mathrm{~mA}$. The output wavelength is coarsely adjusted in the vicinity of the cesium $\mathrm{D}_{2}$ resonance line $(852.1 \mathrm{~nm})$ by changing the temperature, and finely tuned to one of the hyperfine components by varying the injection current [5].

The experimental set-up is shown in figure 1. In region $\mathrm{A}$, the narrowband, linearly polarized laser radiation produces, through optical pumping, a population difference between the hyperfine Zeeman sublevels $F=3, m_{F}=0$ and $F=4, m_{F}=0$ of the cesium ground state [6]. The atomic beam fluorescence is used to stabilize and lock the laser frequency [4] to a specific hyperfine component of the $D_{2}$ line 


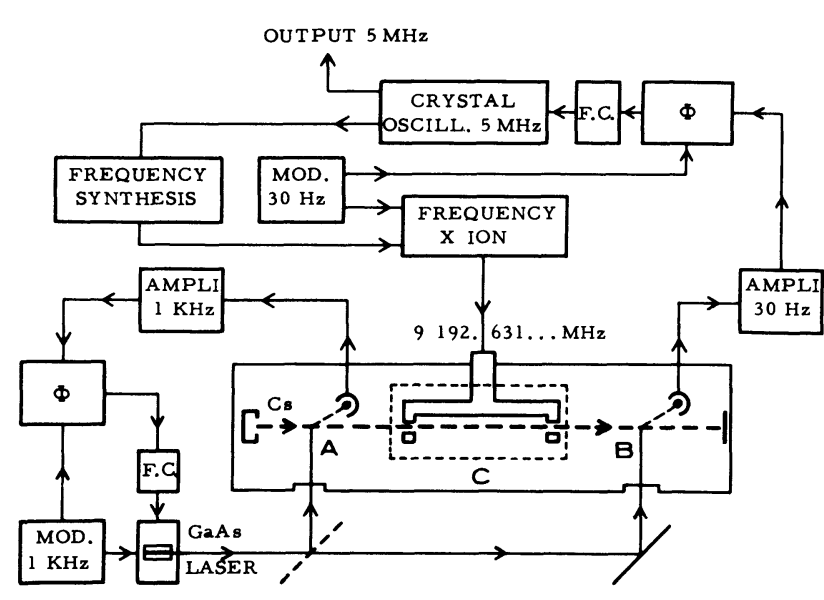

$\Phi$ : PHASE DETECTION

F.C. : FREQUENCY CONTROL

Fig. 1. - Schematics of a cesium beam atomic clock optically pumped with a laser (here, a GaAs diode laser).

$\left(6{ }^{2} \mathrm{~S}_{1 / 2} \leftrightarrow 6{ }^{2} \mathrm{P}_{3 / 2}\right)$. Optical pumping efficiency is found larger with the hyperfine transitions

$$
F=3 \leftrightarrow F^{\prime}=3 \text { or } F=4 \leftrightarrow F^{\prime}=4
$$

than with the others (Fig. 2). In region C, the transition between the levels $m_{F}=0$ is induced by excitation of a Ramsey-type resonant cavity at $9192.631 \ldots \mathrm{MHz}$. The resulting saturation between the populations of the $m_{F}=0$ levels produces, in turn, a change in the intensity of the laser-induced fluorescence of the atomic beam in region $B$.

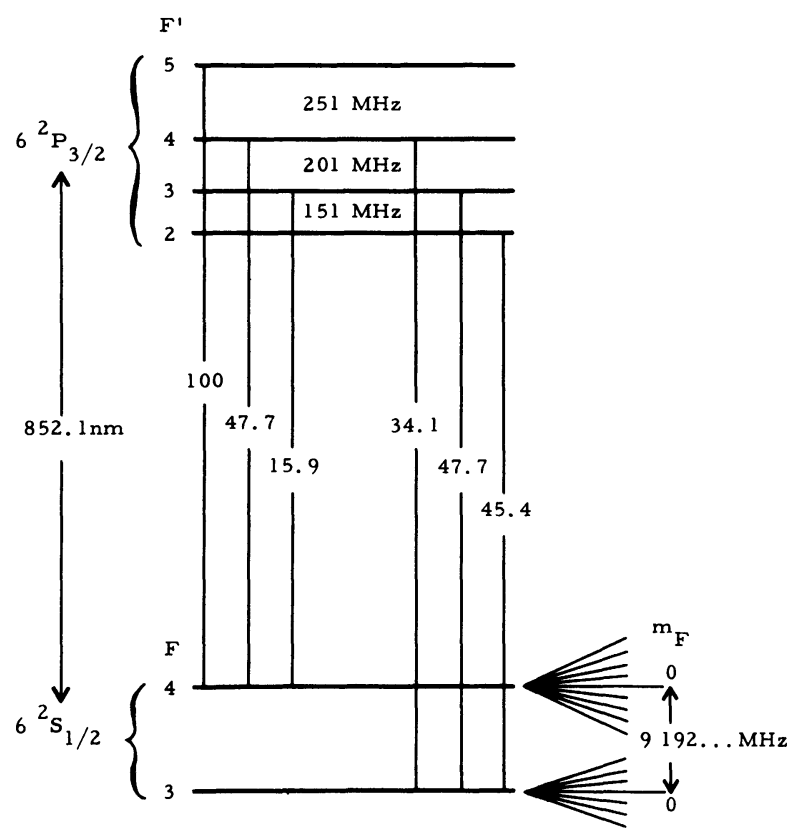

Fig. 2. - Relevant energy levels (not to scale) in the cesium $D_{2}$ resonance line used for optical pumping. The numbers assigned to the various hyperfine lines represent the relative transition probabilities.

The microwave excitation signal is generated through a multiplier chain from a $5 \mathrm{MHz}$ crystal oscillator. This signal is frequency modulated, at a low rate and with a small frequency excursion, thus yielding a low-frequency modulation of the fluorescence signal in the $\mathbf{B}$ region. By comparing in a phase detector the phase of this signal with that of the modulation oscillator, a D.C. error signal is obtained, positive or negative, depending on which side of the hyperfine resonance the microwave excitation frequency is located. This error signal is fed back in proper phase, through an amplifier and integrator, to an element controlling the frequency of the crystal oscillator. Therefore, the crystal-oscillator frequency is locked to the ${ }^{133} \mathrm{Cs}$ hyperfine resonance and a frequency standard, or atomic clock, having a very long-term stability is realized.

Figure 3 shows a recording of the derivative of the microwave Ramsey pattern obtained with a $22.5 \mathrm{~cm}$ long resonant cavity. Shown also is a graph of the derivative of the theoretical lineshape $\left\langle P_{0-0}(\omega)\right\rangle$, obtained by averaging the transition probability [7]

$$
P_{0-0}(\omega)=\sin ^{2} \frac{\Omega l}{v} \cos ^{2} \frac{\left(\omega-\omega_{0}\right) L}{2 v}
$$

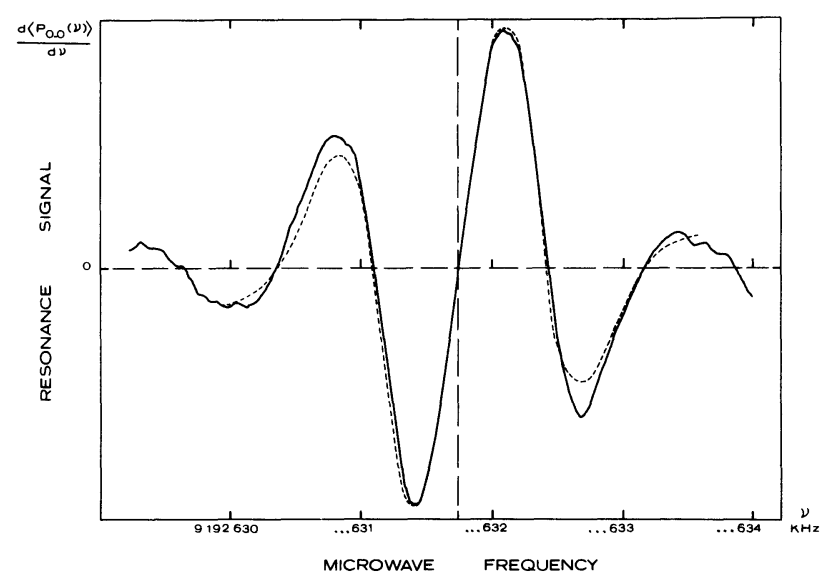

Fig. 3. - Ramsey pattern observed for the $F=3, m_{F}=0 \leftrightarrow F=4$, $m_{F}=0$ microwave transition (derivative curve). The dotted line is the derivative of the theoretical lineshape, calculated for the following values of the parameters $: l=1 \mathrm{~cm}, L=22.5 \mathrm{~cm}$, $\alpha=200 \mathrm{~m} / \mathrm{s}, \Omega l / \alpha=1.885$.

over the velocity distribution $f(v)$ of the atoms in the beam. In expression (1), valid for $\left|\omega-\omega_{0}\right| \ll \Omega$, $\omega=2 \pi v$ is the circular frequency of the microwave field, $\omega_{0}$ the atomic resonance frequency, $\Omega$ the Rabi nutation frequency (proportional to the field amplitude), $l$ the length of the interaction regions of the atoms with the field, $L$ the distance between these two regions and $v$ the atomic velocity. The average has been performed over the usual modified-Maxwellian velocity distribution of effusive beams, $f(v)=2\left(v^{3} / \alpha^{4}\right) \exp \left(-v^{2} / \alpha^{2}\right)$, here taken with the most probable velocity $\alpha=200 \mathrm{~m} / \mathrm{s}$. The agreement between the theoretical and experimental curves is seen to be quite good. This means that, in this experiment, there is no narrowing of the Ramsey pattern 
as in the rubidium experiment [3], where optical pumping with a spectral lamp enhanced the contribution of the slow atoms to the overall signal. This is easily understood if one considers that the spectral density of the monochromatic laser radiation is here about $10^{3}$ times higher than for a spectral lamp, so that the efficiency of optical pumping is about the same for all the atoms, independently of their transit time across the light beam. However, the lack of the slight resonance-narrowing is more than compensated by the large increase in the signal intensity which is provided by the laser.

Some preliminary tests have been made of the apparatus operated as an atomic clock. To this end, the frequency of the oscillator, locked to the centre of the Ramsey pattern (using the discriminant signal shown in Fig. 3), was compared to the frequency of a standard commercial cesium beam clock. The static magnetic field $H_{0}$ in the microwave cavity was changed through a calibrated field coil, and the corresponding frequency shifts were measured, checking the known relation :

$$
v=9192631770 \mathrm{~Hz}+427 H_{0}^{2}
$$

(with $H_{0}$ in gauss) to an accuracy of a few parts in $10^{11}$.

The good signal-to-noise ratio obtained in this experiment suggests that, with improved electronics for detection and servocontrol, a much better accuracy and stability could be achieved. Also, diode lasers are now being available for C.W. operation at room temperature with lifetimes exceeding $10^{4}$ hours, and this should allow the realization of even more practical devices than the laboratory apparatus just described.

Acknowledgments. - We would like to thank Professor A. Kastler, who initiated this experiment, for his encouragement and advice during the course of the work.

\section{References}

[1] Glaze, D. J., Hellwig, H., Allan, D. W. and Jarvis, S., Jr., Metrologia 13 (1977) 17.

[2] Kastler, A., J. Physique Radium 11 (1950) 255.

[3] Cérez, P., Arditi, M. and Kastler, A., C.R. Hebd. Séan. Acad. Sci. 267 (1968) 282;

ARditi, M. and CéreZ, P., I.E.E.E. Trans. Instrum. Meas. IM-21 (1972) 391 ;

Cérez, P. and Hartmann, F., I.E.E.E. J. Quantum Electron. QE-13 (1977) 344.
[4] Picqué, J. L., Metrologia 13 (1977) 115.

[5] Picqué, J. L., Roizen, S., Stroke, H. H. and Testard, O., Appl. Phys. 6 (1975) 373;

Picqué, J. L. and Roizen, S., Appl. Phys. Lett. 27 (1975) 340.

[6] Arditi, M., Hirano, I. and Tougne, P., J. Phys. D 11 (1978) 2465 ;

Hirano, I. and Arditi, M., Opt. Commun. 26 (1978) 66.

[7] Ramsey, N. F., Molecular Beams (Clarendon Press, Oxford) 1969, p. 124. 